\title{
Fermi acceleration on the annular billiard
}

\author{
R. Egydio de Carvalho, F. Caetano Souza, and Edson D. Leonel \\ Departamento de Estatística, Matemática Aplicada e Computação, Instituto de Geociências e Ciências Exatas, \\ Universidade Estadual Paulista, UNESP, Avenida 24A, 1515-Bela Vista, 13506-700, Rio Claro, São Paulo, Brazil
}

(Received 16 February 2006; published 30 June 2006; corrected 6 July 2006)

\begin{abstract}
We study the phenomenon of unlimited energy growth for a classical particle moving in the annular billiard. The model is considered under two different geometrical situations: static and breathing boundaries. We show that when the dynamics is chaotic for the static case, the introduction of a time-dependent perturbation allows that the particle experiences the phenomenon of Fermi acceleration even when the oscillations are periodic.
\end{abstract}

DOI: 10.1103/PhysRevE.73.066229

PACS number(s): 05.45.Pq, 05.45.Gg

\section{INTRODUCTION}

The phenomenon of Fermi acceleration was noticed for the first time in 1949 [1] when Enrico Fermi attempted to describe the mechanism in which cosmic charged particles could be accelerated by stochastic time-dependent magnetic clouds. This mechanism is basically associated with a classical particle subjected to a time-dependent perturbation in a nonlinear context. After that many other different versions of the original one-dimensional Fermi accelerator model were proposed and studied. One of them, known as the FermiUlam model (FUM), consists of a particle moving between two rigid walls, one fixed and the other periodically time dependent [2]. The results obtained showed that the acceleration was not observed. Ulam's results were explained by Zaslavsky and Chirikov [3], Lieberman and Lichtenberg [4], and Brahic [5]. They showed that for smooth wall oscillation, the stochastic mechanism worked but the energy growth was limited by invariant spanning curves present on the corresponding phase space. On the other hand, for the case where the wall oscillation is not smooth enough, then those barriers did not exist anymore. A very similar version of this problem, also known as the bouncer model, consisting of a particle bouncing vertically in a periodically oscillating wall, was considered under the effect of a constant gravitational field [6-8]. It was shown that depending on the combinations of control parameters and initial conditions, the particle might gain energy unlimitedly. The difference between the two models (FUM and bouncer) was latter explained by Lichtenberg, Lieberman, and Cohen [9]. In the context of onedimensional time-dependent systems, many models concerning Fermi acceleration have been made. It is worth emphasizing three of them: the square well $[10,11]$, the step potential barrier [12], and a hybrid version of the FUM [13]. For these models, Fermi acceleration was observed only when the perturbation was stochastic.

On the other hand, Loskutov, Ryabov, and Akinshin have considered a Lorentz-type dispersing billiard whose boundary oscillations were periodic and also stochastic in time [14]. Their calculations showed that Fermi acceleration had taken place in both situations. In the stochastic case they have also identified two basic acceleration mechanisms. The first one comes from the first moment which drives all particles to higher velocities, while the second one comes from fluctuations and is controlled by two conditions: (i) the posi- tiveness of the second moment and (ii) a positive asymmetry for the velocities. In that context, chaotic dynamics brought a new approach for kinetic description of physical systems, due to the fractal, multifractal, and stickiness structures of the phase space. As a consequence, fractional kinetics and anomalous transport may arise in chaotic systems [15-19]. Brief, historical comments on Fermi acceleration linking it with fractional kinetics can be found in Ref. [20]. Recently the same authors of Ref. [14], studying dynamical properties of stadium billiards [21], under periodic time-dependent boundaries, announced a conjecture (the LRA conjecture) saying that a chaotic dynamics for a billiard with static boundary is a sufficient condition for the Fermi acceleration in the system when a boundary perturbation is introduced. Later, Loskutov and Ryabov [22] continued their studies on the stadiumlike billiards. The question posed initially by Ulam naturally returns, the study of the Fermi mechanism in systems with time-periodic perturbations. In that context we revisit, in this paper, the problem of a particle bouncing elastically in the annular billiard but now with the circles breathing periodically.

Thus, our purpose is to investigate the effect of the timedependent boundaries on the dynamics, especially concerning the LRA conjecture. The main reasons that we have chosen the annular billiard as our model are: (i) it is very familiar to us [23-25] and (ii) it has been used in many different investigations including experimental works [26-29]. In addition, we have studied a simplified version of this model [30] as an attempt to verify the LRA conjecture. The geometrical situation is such that it is possible to have analytically both the concentric and the eccentric maps. The dynamics runs in the free space in the ring between the two circles and the particle suffers elastic collisions with both boundaries. Initially, we consider the static case and we present the area-preserving maps that describe the full dynamics. Next we introduce a periodically varying perturbation for both boundaries. The discrete maps are obtained analytically and they contain many parameters. In order to investigate Fermi acceleration in the context of periodic oscillations, we vary only a few parameters in specific ranges.

The paper is organized as follows. In Sec. II we present and discuss some representative numerical results for the static case of the annular billiard. In Sec. III we deal with the time-dependent boundaries and we obtain the expressions of the maps. In Sec. IV we present and discuss the numerical results. The conclusion and the final remarks are presented in Sec. V. 


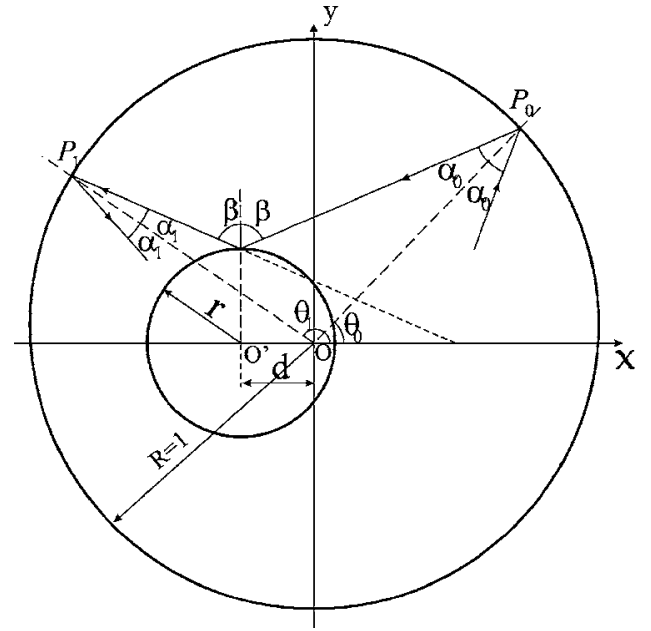

FIG. 1. A geometric scheme of a particle in the static annular billiard.

\section{THE STATIC BILLIARD}

We describe in this section the dynamics of the annular billiard with static boundaries. The system consists of a classical particle confined in an annular region limited by two circumscribed circles, which we will then refer to as the annulus. The geometry may either include the concentric or eccentric configurations $[23,31]$. We define the radius of the outer circle as $R=1$, the radius of the inner circle as $r$, and the eccentricity as $(d)$ (see Fig. 1 for a pictorial example of the annular billiard). We use the constraint $r+d<1$, which implies that the inner circle can never reach the external one.

Inside the annulus, a particle moves freely along a straight line with constant kinetic energy until it collides elastically with the boundaries. After suffering a collision the particle is specularly reflected in the sense that the incidence angle is equal to the reflection angle. The map describing the dynamics is given by $M\left(\theta_{n}, \alpha_{n}\right)=\left(\theta_{n+1}, \alpha_{n+1}\right)$. It is easy to see in Fig. 1 that we can determine the position of the particle on the boundary by the specification of the two angles $\theta$ and $\alpha$. The range of $\alpha$ is $[-\pi / 2, \pi / 2]$ while of $\theta$ is $\theta \in[-\pi, \pi]$.

There are also two kinds of motion which are distinguished by the so called tangency condition [23,31], namely,

$$
\left|\sin \left(\alpha_{n}\right)-d \sin \left(\theta_{n}-\alpha_{n}\right)\right| \leqslant r .
$$

If the combination of both $\left(\theta_{n}, \alpha_{n}\right)$ are such that condition (1) is not matched, then we have the dynamics described by map $M_{A}$, and therefore we have a movement of type $A$.

Type A. Between two successive impacts with the external boundary, the particle does not collide with the internal circle and the map $M_{A}$ is

$$
M_{A}:\left\{\begin{array}{l}
\alpha_{n+1}=\alpha_{n} \\
\theta_{n+1}=\pi+\theta_{n}-2 \alpha_{n} .
\end{array}\right.
$$

On the other hand, movements of type $B$ are those obtained when condition (1) is satisfied, i.e., see the following.

Type $B$. After suffering a collision with the external boundary, the particle hits elastically with the internal circle and then it reaches the external circle again.
The map $M_{B}$ is given by

$$
M_{B}:\left\{\begin{array}{l}
\alpha_{n+1}=\arcsin \left(r \sin \beta-d \sin \theta_{a}\right) \\
\theta_{n+1}=-\alpha_{n+1}+2 \beta+\theta_{n}-\alpha_{n},
\end{array}\right.
$$

where

$$
\begin{gathered}
\theta_{a}=\theta_{n+1}+\alpha_{n+1}=2 \beta+\theta_{n}-\alpha_{n}, \\
\beta=\arcsin \left\{\frac{1}{r}\left[\sin \alpha_{n}-d \sin \left(\theta_{n}-\alpha_{n}\right)\right]\right\} .
\end{gathered}
$$

If we set $d=0$ (concentric case), the phase space for both $r=0$ and $r \neq 0$ are filled by straight lines parallel to the $\theta$ axis. The system is then integrable and such integrability appears due to the angular momentum preservation with respect to the origin of coordinate system $O$. The set of plots presented in Fig. 2 shows the role of the eccentricity in the increasing of the chaotic sea. We emphasize that for all the figures, the corresponding Whispering Gallery Orbits (WGO), referent to $|\sin (\alpha)| \geqslant r+d=0.80$, are not shown. They consist of straight lines parallel to the $\theta$ axis.

The nonintegrability of the eccentric case is related to the break of the angular momentum preservation and as consequence, a very rich structure of periodic orbits and resonances arises [32,33].

\section{THE TIME-DEPENDENT BILLIARD}

We discuss in this section the dynamics of a particle on the annular billiard with time-varying boundaries. The time perturbation is chosen in such way that both the internal and the external boundaries oscillate simultaneously and preserve their concavities. In this sense, the new radii are

$$
\begin{gathered}
R^{*}\left(t^{\prime}\right)=1+\epsilon_{R} \cos \left(\omega_{R} t^{\prime}+\varphi_{0}\right), \\
r^{*}\left(t^{\prime}\right)=r+\epsilon_{r} \cos \left(\omega_{r} t^{\prime}+\phi_{0}\right),
\end{gathered}
$$

where $\left(\epsilon_{r}, \omega_{r}, \phi_{0}\right)$ and $\left(\epsilon_{R}, \omega_{R}, \varphi_{0}\right)$ are the amplitude of oscillation, the frequency of oscillation and the initial phases, respectively, for both the internal and external boundaries. Moreover, we can introduce dimensionless and more convenient variables. We begin defining a new time $t=\omega_{R} t^{\prime}$ so that we have

$$
\begin{aligned}
& R(t)=1+\epsilon_{R} \cos \left(t+\varphi_{0}\right), \\
& r(t)=r+\epsilon_{r} \cos \left(\omega t+\phi_{0}\right),
\end{aligned}
$$

where $\omega=\omega_{r} / \omega_{R}, R(t)=R^{*}\left(t / \omega_{R}\right)$, and $r(t)=r^{*}\left(t / \omega_{R}\right)$. According to the new set of control parameters, the expressions for both velocities of the boundaries are

$$
\begin{gathered}
v_{R}(t)=\frac{d R(t)}{d t}=-\epsilon_{R} \sin \left(t+\varphi_{0}\right), \\
v_{r}(t)=\frac{d r(t)}{d t}=-\epsilon_{r} \omega \sin \left(\omega t+\phi_{0}\right) .
\end{gathered}
$$

As the dynamics evolves, the particle can gain or loose 

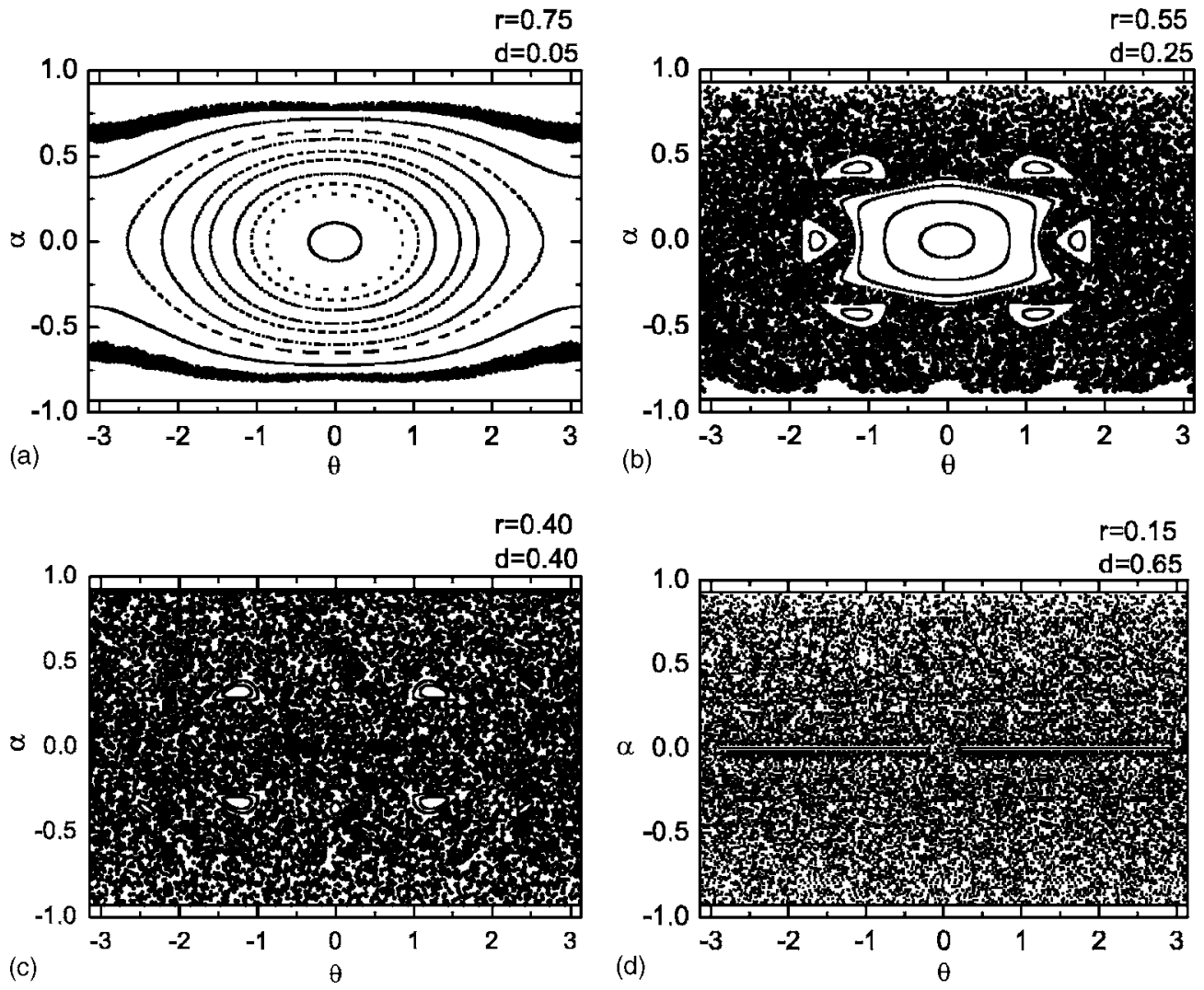

FIG. 2. Phase space for the eccentric case. The control parameters used were (a) $r=0.75, d=0.05$, (b) $r=0.55, d=0.25$, (c) $r=0.40$, $d=0.40$, and (d) $r=0.15, d=0.65$.

energy as it hits the boundaries. What call the collision zone is the region in the billiard plane where the particle may hit the boundaries. For the external boundary, the collision zone is defined as $\left(1-\epsilon_{R}\right) \leqslant R \leqslant\left(1+\epsilon_{R}\right)$ and for the internal boundary it is $\left(r-\epsilon_{r}\right) \leqslant R \leqslant\left(r+\epsilon_{r}\right)$ (see Fig. 3). The dynamics is described using a nonlinear discrete map, $T\left(\theta_{n}, \alpha_{n}, v_{n}, \varphi_{n}, \phi_{n}\right)=\left(\theta_{n+1}, \alpha_{n+1}, v_{n+1}, \varphi_{n+1}, \phi_{n+1}\right)$, which gives all the relevant information for the dynamics between the collision $n$th and $(n+1)$ th with the external boundary. The variables $\theta_{n}$ and $\alpha_{n}$ are the angles previously used, $v_{n}$ is

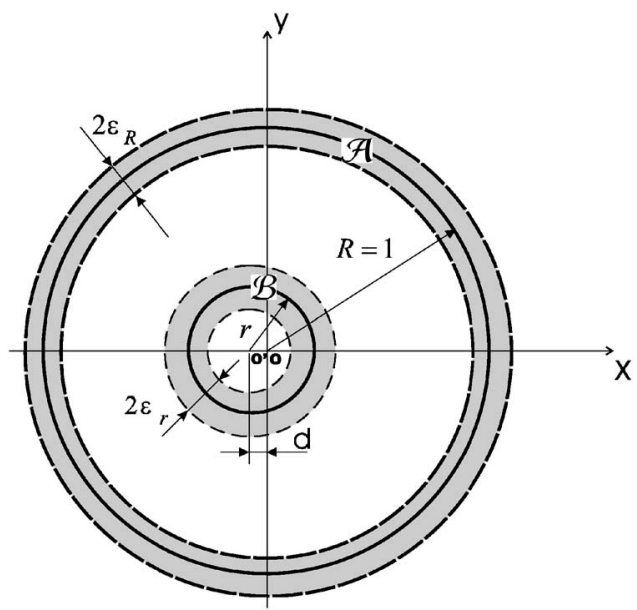

FIG. 3. A pictorial scheme for the annular billiard with time varying boundaries. the velocity, and $\left(\varphi_{n}, \phi_{n}\right)$ are, respectively, the phases of both time-varying boundaries.

For the derivation of the map, we suppose that the particle is initially located in the external collision zone whose rectangular Cartesian coordinates are given by

$$
\begin{aligned}
& x_{n}=R\left(\varphi_{n}\right) \cos \left(\theta_{n}\right), \\
& y_{n}=R\left(\varphi_{n}\right) \sin \left(\theta_{n}\right),
\end{aligned}
$$

with $R\left(\varphi_{n}\right)$ given by Eq. (2) for the initial time $t=t_{n}$, where the index $n$ denotes the $n$th iteration. We use the conventions $\varphi_{n}=t_{n}+\varphi_{0} \bmod 2 \pi, \phi_{n}=\omega t_{n}+\phi_{0} \bmod 2 \pi$. There are three different physical situations for the dynamics between the collisions. We begin considering the case for which the particle suffers successive collisions with the external boundary without leaving the external collision zone.

Case 1. In this case, the particle experiences multiples collisions with the external boundary before leaving the external collision zone. The dynamics is illustrated in Fig. 4. The rectangular Cartesian components for the position of the particle are

$$
\begin{aligned}
& x_{p}(t)=x_{n}+v_{n x} t, \\
& y_{p}(t)=y_{n}+v_{n y} t,
\end{aligned}
$$

where $v_{n x}=v_{n} \cos \left(\theta_{n}\right)$ and $v_{n y}=v_{n} \sin \left(\theta_{n}\right)$ are the corresponding components of the velocity $v_{n}$. We define a radius for the 


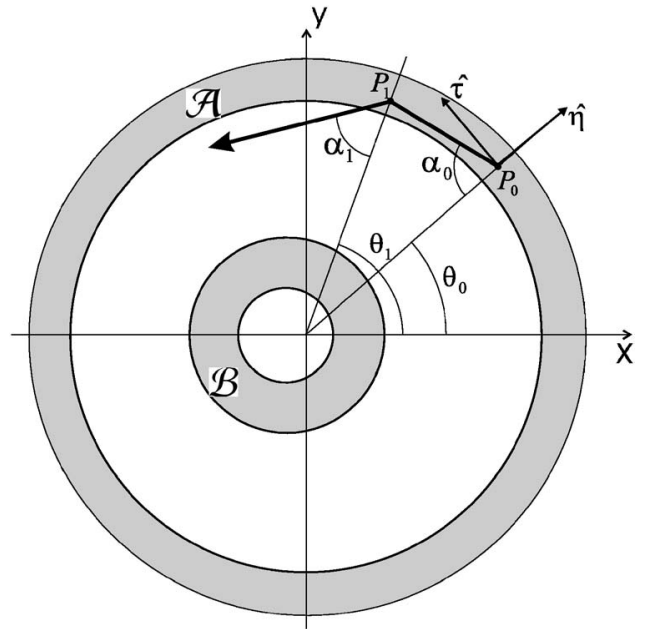

FIG. 4. Illustration of the successive collisions for case 1 . The particle begins in the point $P_{0}$ and hits the circle in $P_{1}$ without leaving the collision zone.

position of the particle as $R_{p}(t)=\sqrt{x_{p}^{2}(t)+y_{p}^{2}(t)}$. Using Eqs. (5) and (6), we obtain

$$
R_{p}(t)=\sqrt{R_{n}^{2}+2\left(x_{n} v_{n x}+y_{n} v_{n y}\right) t+v_{n}^{2} t^{2}},
$$

where $R_{n}=\sqrt{x_{n}^{2}+y_{n}^{2}}$ and $v_{n}=\sqrt{v_{n x}^{2}+v_{n y}^{2}}$. The mixed term in Eq. (7) can be written in a more appropriate form. Note that it represents the scalar product between $\left(x_{n}, y_{n}\right) \cdot\left(v_{n x}, v_{n y}\right)$ $=x_{n} v_{n x}+y_{n} v_{n y}$. Representing such a product in terms of polar coordinates, we obtain $\left(R_{n}, 0\right)\left(v_{n \eta}, v_{n \tau}\right)=R_{n} v_{n \eta}[1$ $\left.+\epsilon_{R} \cos \left(\varphi_{n}\right)\right] v_{n \eta}$, where $v_{n \eta}=-v_{n} \cos \left(\alpha_{n}\right)$ is the normal velocity component while $v_{n \tau}=v_{n} \sin \left(\alpha_{n}\right)$ is the tangential one. Incorporating these modifications, the expression for $R_{p}(t)$ is given by

$$
R_{p}(t)=\sqrt{\left[1+\epsilon_{R} \cos \left(\varphi_{n}\right)\right]^{2}+2 v_{n \eta}\left[1+\epsilon_{R} \cos \left(\varphi_{n}\right)\right] t+v_{n}^{2} t^{2}} .
$$

As an attempt to obtain the instant of the impact of the particle with the external boundary, we match the condition $R_{p}(t)=R(t)$. We thus define the function,

$$
\begin{aligned}
g(t)= & {\left[1+\epsilon_{R} \cos \left(t+\varphi_{n}\right)\right]^{2}-\left[1+\epsilon_{R} \cos \left(\varphi_{n}\right)\right]^{2} } \\
& -2 v_{n \eta}\left[1+\epsilon_{R} \cos \left(\varphi_{n}\right)\right] t-v_{n}^{2} t^{2} .
\end{aligned}
$$

If $g(t)$ admits roots in the interval $t \in(0,2 \pi]$, then we take the smaller one denoting it as $t_{c}$. If in the interval $0<t \leqslant t_{c}$ the condition

$$
R_{p}(t) \geqslant\left(1-\epsilon_{R}\right)
$$

is always true, then the time $t_{n+1}=t_{n}+t_{c}$ represents the instant of the $(n+1)$ th impact between the particle and the external circle. If no solution for $g(t)=0$ is found, or if condition (8) is not matched, then case 2 or 3 applies. Evaluating then the variables for the instant $t_{n+1}$, we obtain

$$
\theta_{n+1}=\arctan \left[\frac{y_{p}\left(t_{n+1}\right)}{x_{p}\left(t_{n+1}\right)}\right] .
$$

Immediately before the collision, the new components of the velocity are

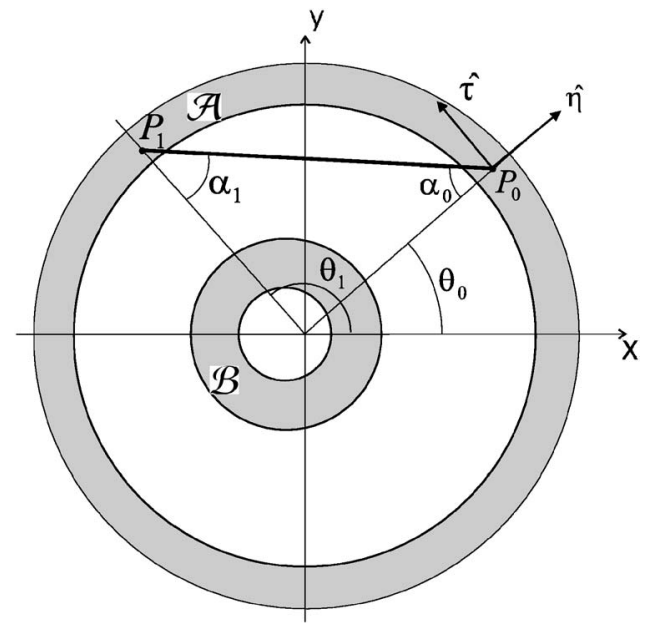

FIG. 5. Illustration for case 2. The particle leaves $A$ without crossing $B$.

$$
\begin{gathered}
v_{n \eta}=v_{n x} \cos \left(\theta_{n+1}\right)+v_{n y} \sin \left(\theta_{n+1}\right), \\
v_{n \tau}=-v_{n x} \sin \left(\theta_{n+1}\right)+v_{n y} \cos \left(\theta_{n+1}\right),
\end{gathered}
$$

After the impact, only the radial component of the velocity is modified, so that we obtain

$$
\begin{gathered}
v_{(n+1) \eta}=-v_{n \eta}+2 v_{R}\left(t_{n+1}\right), \\
v_{(n+1) \tau}=v_{n \tau},
\end{gathered}
$$

where $v_{R}\left(t_{n+1}\right)$ is given by Eq. (3). The respective expressions for the new velocity, the new incidence angle, and corresponding phases are

$$
\begin{gathered}
v_{n+1}=\sqrt{v_{(n+1) \eta}^{2}+v_{(n+1) \tau}^{2}}, \\
\alpha_{n+1}=\arctan \left[-\frac{v_{(n+1) \tau}}{v_{(n+1) \eta}}\right], \\
\varphi_{n+1}=\varphi_{n}+t_{c} \bmod 2 \pi, \\
\phi_{n+1}=\phi_{n}+\omega t_{c} \bmod 2 \pi .
\end{gathered}
$$

We now discuss case 2 .

Case 2. In this case, the particle leaves the external collision area without, however, crossing the internal collision area. After leaving the external collision area (see region $A$ of Fig. 5), the coordinates $\left(\alpha_{n}, \theta_{n}\right)$ allow us to check the tangency condition

$$
\left|R_{n} \sin \left(\alpha_{n}\right)-d \sin \left(\theta_{n}-\alpha_{n}\right)\right| \leqslant\left(r+\epsilon_{r}\right),
$$

where $R_{n}=1+\epsilon_{R} \cos \left(\varphi_{n}\right)$. If the tangency condition above is not satisfied, then we proceed in case 2; otherwise case 3 applies. Between the impacts, the particle travels in a straight line and we can obtain an analytic expression for the time traveled after the $n$th impact until the particle enters the external collision area again. We have to obtain the intersection of the particle's radius $R_{p}(t)$ with the lower limit of the ex- 
ternal collision area $\left(1-\epsilon_{R}\right)$. Such condition give rises to the following equation:

$$
\begin{gathered}
v_{n}^{2} t^{2}+2 v_{n \eta}\left[1+\epsilon_{R} \cos \left(\varphi_{n}\right)\right] t+\left[1+\epsilon_{R} \cos \left(\varphi_{n}\right)\right]^{2} \\
-\left(1-\epsilon_{R}\right)^{2}=0 .
\end{gathered}
$$

Equation (9) admits two real solutions. The smallest one corresponds to the instant that the particle crosses the radius $\left(1-\epsilon_{R}\right)$ and leaves the external collision area. We are interested in the second solution, which we will call $t_{f}$, that gives the instant when the particle crosses $\left(1-\epsilon_{R}\right)$ toward the collision area. Since the particle is again inside the external collision area, it necessarily suffers an impact with the external boundary. Moreover, this new impact does not characterize a successive collision since the particle has already traveled outside of $\left[\left(1-\epsilon_{R}\right),\left(1+\epsilon_{R}\right)\right]$. We have now to obtain the instant of the impact with the external boundary.

The new coordinates of the position of the particle at the entrance of the external collision zone are

$$
\begin{aligned}
& x_{p}\left(t_{f}\right)=x_{n}+v_{n x} t_{f}, \\
& y_{p}\left(t_{f}\right)=y_{n}+v_{n y} t_{f},
\end{aligned}
$$

where $x_{n}$ and $y_{n}$ are given by Eq. (5) and (6). The components of the particle velocity, in polar coordinates, evaluated in the time $t_{f}$ are

$$
\begin{gathered}
v_{n \eta}=v_{n x} \cos \left(\theta_{f}\right)+v_{n y} \sin \left(\theta_{f}\right), \\
v_{n \tau}=-v_{n x} \sin \left(\theta_{f}\right)+v_{n y} \cos \left(\theta_{f}\right),
\end{gathered}
$$

where

$$
\theta_{f}=\arctan \left[\frac{y_{p}\left(t_{f}\right)}{x_{p}\left(t_{f}\right)}\right] .
$$

As an attempt to obtain the instant of the impact we have to match the condition

$$
R_{p}(t)=R(t),
$$

where $R_{p}(t)$ is given by Eq. (7) but therefore evaluation of $\theta_{f}$ and $R(t)$ is given by Eq. (2). Equation (12) is also rewritten as

$$
\begin{aligned}
f(t)= & {\left[1+\epsilon_{R} \cos \left(t+t_{f}+\varphi_{n}\right)\right]^{2}-\left[1+\epsilon_{R} \cos \left(\varphi_{n}\right)\right]^{2} } \\
& -v_{n}^{2} t^{2}-2 v_{n \eta}\left[1+\epsilon_{R} \cos \left(\varphi_{n}\right)\right] t .
\end{aligned}
$$

The equation above admits more than one solution but we are interested in the smallest. We will call by $t_{c}$ the time between the interval $t \in[0,2 \pi)$. Since the instant of the impact is obtained, the new time is $t_{n+1}=t_{n}+t_{f}+t_{c}$ and the new coordinates of the particle are

$$
\begin{aligned}
& x_{n+1}=x_{p}\left(t_{f}\right)+v_{n x} t_{c}, \\
& y_{n+1}=y_{p}\left(t_{f}\right)+v_{n y} t_{c} .
\end{aligned}
$$

We can now define the new angle,

$$
\theta_{n+1}=\arctan \left[\frac{y_{n+1}}{x_{n+1}}\right],
$$

for which the new components of the velocity, immediately before the collision, are

$$
\begin{aligned}
& v_{n \eta}=v_{n x} \cos \left(\theta_{n+1}\right)+v_{n y} \sin \left(\theta_{n+1}\right), \\
& v_{n \tau}=-v_{n x} \sin \left(\theta_{n+1}\right)+v_{n y} \cos \left(\theta_{n+1}\right)
\end{aligned}
$$

After the collision, only the radial component of the velocity changes, then we have

$$
\begin{gathered}
v_{(n+1) \eta}=-v_{n \eta}+2 v_{R}\left(t_{c}+t_{f}+\varphi_{n}\right), \\
v_{(n+1) \tau}=v_{n \tau},
\end{gathered}
$$

where $v_{R}$ is given by Eq. (3).

The rectangular Cartesian coordinates for the new velocity are

$$
\begin{aligned}
& v_{(n+1) x}=v_{(n+1) \eta} \cos \left(\theta_{n+1}\right)-v_{(n+1) \tau} \sin \left(\theta_{n+1}\right), \\
& v_{(n+1) y}=v_{(n+1) \eta} \sin \left(\theta_{n+1}\right)+v_{(n+1) \tau} \cos \left(\theta_{n+1}\right) .
\end{aligned}
$$

The modulus of $v_{n+1}$ is then $v_{n+1}=\sqrt{v_{(n+1) x}^{2}+v_{(n+1) y}^{2}}$. The new angle $\alpha_{n+1}$ is obtained through

$$
\alpha_{n+1}=\arctan \left[\frac{v_{(n+1) \tau}}{v_{(n+1) \eta}}\right] .
$$

Finally, the corresponding new phase for both the internal and external boundaries is

$$
\begin{gathered}
\varphi_{n+1}=t_{c}+t_{f}+\varphi_{n} \bmod (2 \pi), \\
\phi_{n+1}=\omega\left(t_{c}+t_{f}\right)+\phi_{n} \bmod (2 \pi) .
\end{gathered}
$$

Let us now present and discuss case 3 .

Case 3. For this case, after the particle leaves the external collision area, it necessarily crosses the internal collision zone. It might suffer an impact with the internal boundary or not. For cases 1 and 2, our expressions for the maps describe the dynamics of a particle suffering elastic collisions with one boundary moving periodically in time [34]. Case 3 now considers collisions with one more periodically varying boundary, the internal one. After the collision and depending on the phase of the internal boundary, the particle can gain or loose energy. There are three different situations that we have to consider for the case 3 :

(i) The particle crosses the collision zone $B$ without suffering any collision with the internal boundary.

(ii) The particle collides once and leaves the internal collision zone without suffering any further collision (see Fig. 6).

(iii) The particle suffers successive collisions with the internal boundary.

Let us now discuss case 1. After leaving the external boundary with rectangular Cartesian coordinates $\left(x_{n}, y_{n}\right)$ the particle arrives in the internal collision zone (region $B$ of Fig. 6) with coordinates 


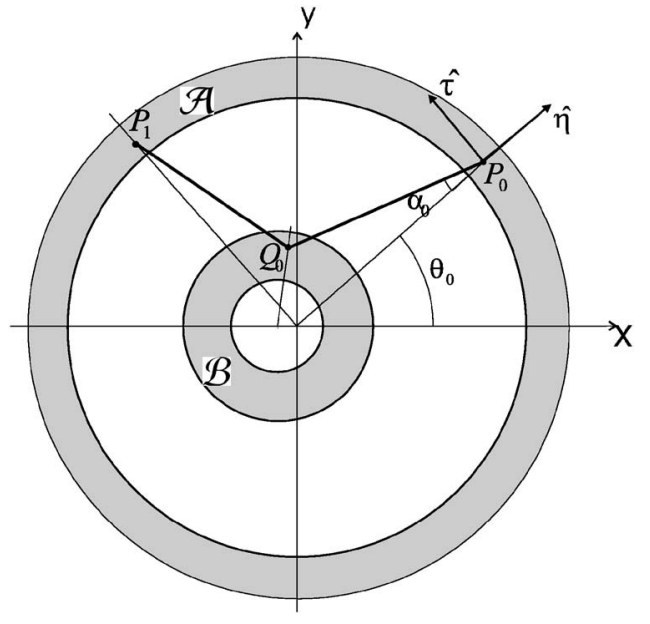

FIG. 6. Illustration for case 3. The particle leaves $A$, collides with the internal boundary in $B$, and finally hits the external boundary in $A$.

$$
\begin{gathered}
x_{b}=r \cos \left(\theta_{b}\right)-d, \\
y_{b}=r \sin \left(\theta_{b}\right),
\end{gathered}
$$

where the angle $\theta_{b}$ is given by

$$
\theta_{b}=\arcsin \left[\frac{R_{n} \sin \left(\alpha_{n}\right)-d \sin \left(\theta_{n}-\alpha_{n}\right)}{r+\epsilon_{r}}\right]+\theta_{n}-\alpha_{n} .
$$

The time spent after the particle leaves the external collision zone and arrives in the internal collision area is given by $t_{f}$ $=\Delta S / v_{n}$. Here,

$$
\Delta S=\sqrt{\left(x_{n}-x_{b}\right)^{2}+\left(y_{n}-y_{b}\right)^{2}},
$$

is the length of the trajectory starting from the point $\left(x_{n}, y_{n}\right)$ up to the entrance in the collision zone $B$ at $\left(x_{b}, y_{b}\right)$. The particle necessarily suffers a collision with the internal boundary if $R_{p}(t)=r(t)$. For this condition, we define the function $f_{r}(t)$ that is written as

$$
\begin{aligned}
f_{r}(t)= & \left(r+\epsilon_{r}\right)^{2}+2\left(r+\epsilon_{r}\right) v_{b \eta} t+v_{n}^{2} t^{2} \\
& -\left(r+\epsilon \cos \left[\omega\left(t+t_{f}\right)+\varphi_{n}\right]\right)^{2},
\end{aligned}
$$

where $v_{b \eta}=v_{n x} \cos \left(\theta_{b}\right)+v_{n y} \sin \left(\theta_{b}\right)$. The situation (1) applies if $f_{r}(t)$ does not have any root in the interval $t \in(0,2 \pi / \omega]$. Then we proceed normally iterating as in the case 2 . If, however, $f_{r}(t)$ has any root, for $t \in(0,2 \pi / \omega]$ we have to deal with situation (2). Calling the solution of Eq. (24) as $t_{r}$, then the coordinates of the particle, measured in the referential frame of the internal boundary, are

$$
\begin{aligned}
& x_{r}^{\prime}=x_{b}^{\prime}+v_{n x} t_{r}, \\
& y_{r}^{\prime}=y_{b}^{\prime}+v_{n y} t_{r},
\end{aligned}
$$

where $x_{b}^{\prime}=r \cos \left(\theta_{b}\right)$ and $y_{b}^{\prime}=r \sin \left(\theta_{b}\right)$. So we can now define the angle $\theta_{r}=\arctan \left(y_{r}^{\prime} / x_{r}^{\prime}\right)$ and the corresponding polar coordinates for the velocity of the particle,

$$
\begin{gathered}
v_{n \eta}=v_{n x} \cos \left(\theta_{r}\right)+v_{n y} \sin \left(\theta_{r}\right), \\
v_{n \tau}=-v_{n x} \sin \left(\theta_{r}\right)+v_{n y} \cos \left(\theta_{r}\right) .
\end{gathered}
$$

The new time is $t_{n+1}=t_{n}+t_{f}+t_{r}$ and after the collision only the radial component of the velocity changes. Then we have

$$
\begin{gathered}
v_{(n+1) \eta}=-v_{n \eta}+2 v_{r}\left(t_{n+1}\right), \\
v_{(n+1) \tau}=v_{n \tau},
\end{gathered}
$$

where the velocity $v_{r}\left(t_{n+1}\right)$ is given by Eq. (4). After the collision, the velocity of the particle has the components

$$
\begin{aligned}
& v_{(n+1) x}=v_{(n+1) \eta} \cos \left(\theta_{r}\right)-v_{(n+1) \tau} \sin \left(\theta_{r}\right), \\
& v_{(n+1) y}=v_{(n+1) \eta} \sin \left(\theta_{r}\right)+v_{(n+1) \tau} \cos \left(\theta_{r}\right) .
\end{aligned}
$$

The condition for the particle suffering a successive collision with the internal boundary is obtained using $R_{p}(t)$ $=r(t)$, and we can define a function $g_{r}(t)$ as

$$
\begin{aligned}
g_{r}(t)= & {\left[r+\epsilon_{r} \cos \left(\omega\left[t_{f}+t_{r}\right]+\phi_{n}\right)\right]^{2} } \\
& +2 v_{(n+1) \eta}\left[r+\epsilon_{r} \cos \left(\omega\left[t_{f}+t_{r}\right]+\phi_{n}\right)\right] t+v_{n+1}^{2} t^{2} \\
& -\left[r+\epsilon_{r} \cos \left(\omega\left[t+t_{f}+t_{r}\right]+\phi_{n}\right)\right]^{2} .
\end{aligned}
$$

If $g_{r}(t)$ does not have any root for $t \in(0,2 \pi / \omega]$, then it is easy to conclude that the particle left the collision zone $B$ (see Fig. 6). If the particle leaves $B$, then we have to obtain the time that it crosses the external collision area $A$, which has the radius $R=\left(1-\epsilon_{R}\right)$. The instant of the crossing, we call it $t_{c}$, corresponds to the largest solution of the following equation:

$$
\begin{gathered}
{\left[v_{(n+1) x}^{2}+v_{(n+1) y}^{2}\right] t^{2}+2\left(x_{r}^{\prime} v_{(n+1) x}+y_{r}^{\prime} v_{(n+1) y}\right) t} \\
+\left(x_{r}^{\prime 2}+y_{r}^{\prime 2}\right)-\left(1-\epsilon_{R}\right)^{2}=0 .
\end{gathered}
$$

The next step is then to iterate normally Eqs. (10), (11), and (13), and then finally Eqs. (14)-(23).

Suppose there exists a time $t_{s}$ for which $g_{r}\left(t_{s}\right)=0$ for $t_{s}$ $\in(0,2 \pi / \omega]$. This necessarily characterizes a successive collision with the internal moving boundary and situation 3 applies. The new coordinates of the particle are

$$
\begin{aligned}
& x_{s}^{\prime}=x_{r}^{\prime}+v_{(n+1) x} t_{s}, \\
& y_{s}^{\prime}=y_{r}^{\prime}+v_{(n+1) y} t_{s} .
\end{aligned}
$$

Immediately before the impact, the components of the particle velocity are

$$
\begin{gathered}
v_{n \eta}^{s}=v_{(n+1) x} \cos \left(\theta_{s}\right)+v_{(n+1) y} \sin \left(\theta_{s}\right), \\
v_{n \tau}^{s}=-v_{(n+1) x} \sin \left(\theta_{s}\right)+v_{(n+1) y} \cos \left(\theta_{s}\right),
\end{gathered}
$$

with the angle $\theta_{s}=\arctan \left(y_{s}^{\prime} / x_{s}^{\prime}\right)$. The upper index $s$ denotes a successive collision. After the collision, the new time is $t_{n+1}=t_{n}+t_{f}+t_{r}+t_{s}$ and the new components of the velocity are

$$
v_{(n+1) \eta}^{s}=-v_{n \eta}^{s}+2 v_{r}\left(t_{n+1}\right),
$$



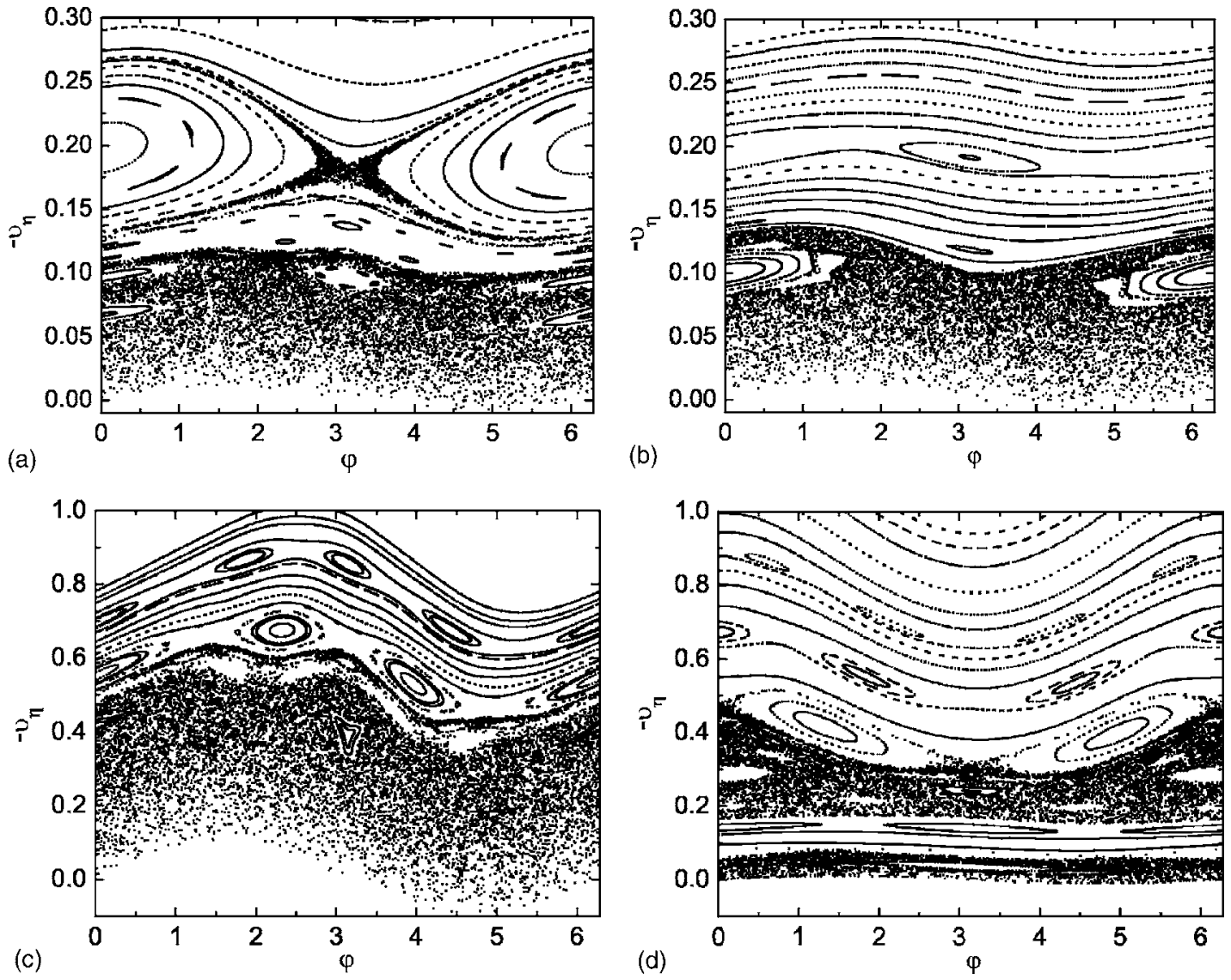

FIG. 7. Phase space in variables $v_{\eta} \times \varphi$ for the concentric case. The control parameters and initial conditions used were $r=0.4, d=0, \omega=1$ and (a) $\epsilon_{R}=\epsilon_{r}=0.01, l=0.01$, and $\varphi_{n}-\phi_{n}=0$; (b) $\epsilon_{R}=\epsilon_{r}=0.01, l=0.01$, and $\varphi_{n}-\phi_{n}=\pi$; (c) $\epsilon_{R}=0.1, \epsilon_{r}=0.01, l=0.1$, and $\varphi_{n}-\phi_{n}=0$; and (d) $\epsilon_{R}=0.01, \epsilon_{r}=0.1, l=0.1$, and $\varphi_{n}-\phi_{n}=0$.

$$
v_{(n+1) \tau}^{s}=v_{n \tau}^{s},
$$

with $v_{r}$ given by Eq. (4). With these new values, we check the condition $g_{r}(t)=0$. If no root is found for $t \in(0,2 \pi / \omega]$, then the particle left the internal zone and it will certainly arrive at the external collision zone. The procedures for this case are the same as for case 2 for the particle entering into the external collision zone. However, if a root is found, the particle is suffering a further and successive collision. We thus make $x_{r} \rightarrow x_{s}, y_{r} \rightarrow y_{s}, v_{(n+1) \eta} \rightarrow v_{(n+1) \eta}^{s}, v_{n+1} \rightarrow v_{n+1}^{s}, t_{r}$ $\rightarrow t_{r}+t_{s}$, and Eqs. (26)-(29) are iterated again until the particle leaves the internal collision zone. From there we follow the procedures of case 2 when the particle enters the collision zone $A$.

\section{NUMERICAL RESULTS}

We discuss in this section our results obtained for the boundaries moving periodically in time. The concentric case is firstly considered, and next the results for the eccentric case are presented and discussed.

\section{A. Concentric case}

For the concentric case, the control parameter $d$ assumes the value $d=0$. To iterate the map we have to give the initial conditions, $\left(\theta_{n}, v_{n}, \varphi_{n}, \phi_{n}\right)$. We stress, however, that as a consequence of the angular momentum preservation, the initial condition $\alpha_{n}$ is obtained from the equation

$$
\sin \left(\alpha_{n}\right)=\frac{l}{\left[1+\epsilon_{R} \cos \left(\varphi_{n}\right)\right] v_{n}},
$$

where we choose some ranges of values for the angular momentum $l$. For large $l$, the particle hits the internal boundary only in the regime of high energy (large values of velocity). So we set the range of $l$ as $l \in\left[0, \epsilon_{R}\right]$. If $l=0$, our model recovers all the results of the well-known Fermi-Ulam accelerator model $[2,35]$.

We show in Fig. 7 the corresponding phase space on the variables $\left(-v_{\eta}, \varphi\right)$. We emphasize that such a system is near integrable even considering both boundaries moving. In this sense, it is easy to see a chaotic sea at low energy surrounding the Kolmogorov-Arnold-Moser (KAM) resonance islands. The chaotic sea is also limited by a set of invariant torus (also called invariant spanning curves). If the reader is not familiarized with the nomenclature of dynamical systems, we suggest the textbooks $[35,36]$. The control parameters and the initial conditions used to construct Fig. 7 were $r=0.4, d=0, \omega=1$, and: (a) $\epsilon_{R}=\epsilon_{r}=0.01, l=0.01$, and $\varphi_{n}$ $-\phi_{n}=0$; (b) $\epsilon_{R}=\epsilon_{r}=0.01, l=0.01$, and $\varphi_{n}-\phi_{n}=\pi$; (c) $\epsilon_{R}$ 

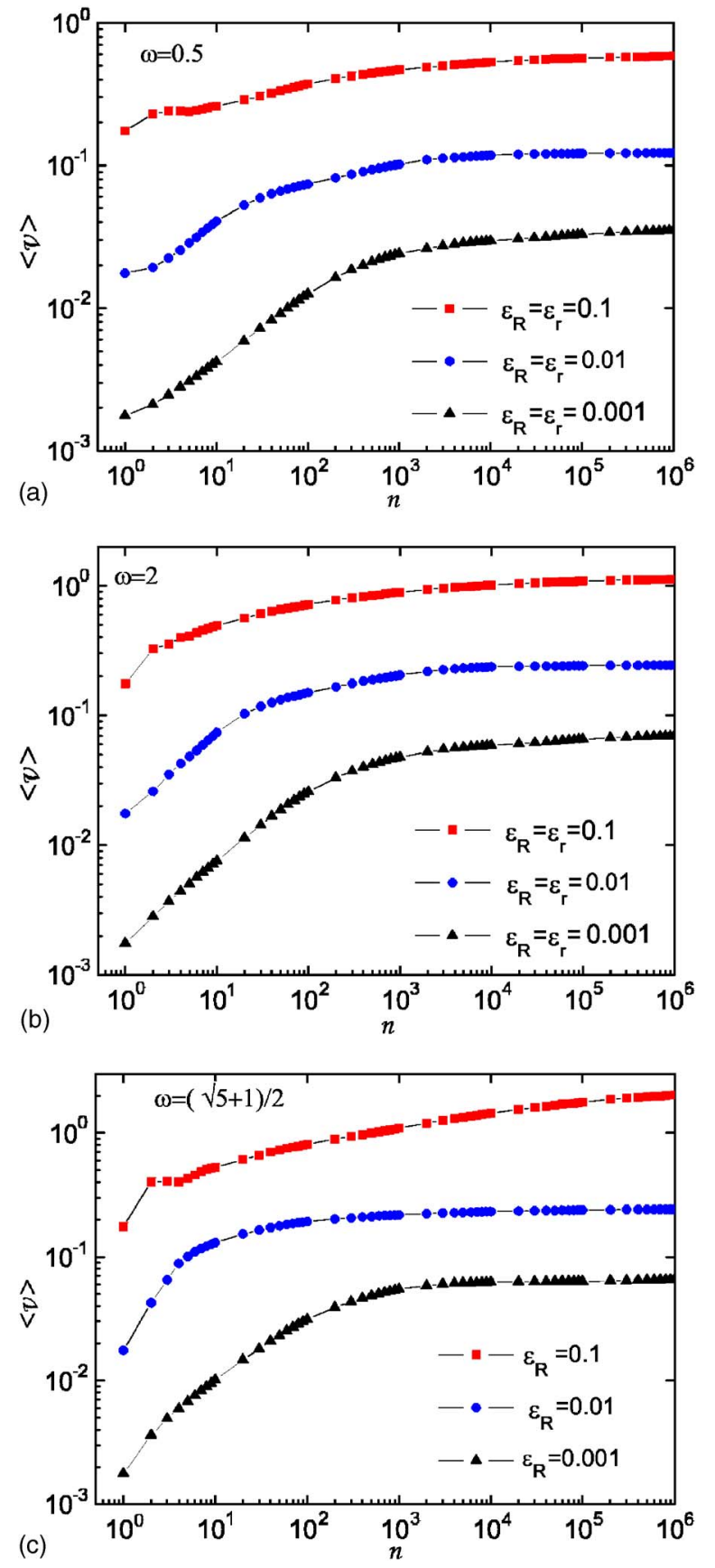

FIG. 8. (Color online) Average velocity as function of the iteration number. The control parameters used were $r=0.5$ and: (a) $\epsilon_{R}$ $=\epsilon_{r}=0.1, \omega=0.5$, and $(\varphi-\phi=0) ;$ (b) $\epsilon_{R}=\epsilon_{r}=0.01, \omega=2$, and $\varphi-\phi$ $=0$; and (c) $\epsilon_{R}=0.001, \epsilon_{r}=\epsilon_{R}(\sqrt{5}+1) / 2$, and $\omega=(\sqrt{5}+1) / 2$.

$=0.1, \epsilon_{r}=0.01, l=0.1$, and $\varphi_{n}-\phi_{n}=0 ;$ and (d) $\epsilon_{R}=0.01, \epsilon_{r}$ $=0.1, l=0.1$, and $\varphi_{n}-\phi_{n}=0$.

It is worth stressing that the invariant spanning curves present in the phase space are responsible for the limited gain of energy. The particle in the chaotic sea might gain or loose energy, but no unlimited Fermi acceleration should be observed. This is numerically verified and the results are shown in Fig. 8. Figure 8 shows the average velocity as a function of the iteration number (i.e., collisions with external boundary). The average is made in an ensemble of $10^{3}$ different initial phases, uniformly distributed in $\varphi \in[0,2 \pi]$. For a small number of iterations, the velocity grows until it reaches a crossover iteration number and from there it bends toward a regime of saturation. This saturation is a consequence of the existence of invariant spanning curves and is consequently none unlimited Fermi acceleration is observed. We would like to emphasize that an analytical approach has been made in the simplified version of the time-dependent annular billiard (see Ref. [30]) in order to obtain the location of the lowest energy invariant spanning curve.

Our results presented and discussed in this section give support to the LRA conjecture [21] in the sense that the dynamics for the concentric static case is entirely integrable. As we shall show, the Fermi acceleration may occur only when the dynamics for the static case is chaotic, as we discuss now.

\section{B. Eccentric case}

Let us now discuss the eccentric case $(d \neq 0)$. We consider two different dynamical scenarios of the static case in order to study the time-dependent billiard. We have fixed the condition $r+d=0.8$ and we choose the configurations (a) $r$ $=0.75$ and $d=0.05$; and (b) $r=0.15$ and $d=0.65$. The reason for choosing such combinations is because the dynamics for the first case is mostly regular around the fixed point $(\theta, \alpha)$ $=(0,0)$ with a small region of instability. On the other hand, the phase space of the second case is predominantly chaotic. The behavior of $\langle v\rangle$ as function of $n$ is shown in Fig. 9.

The averages were made in an ensemble of 500 different initial conditions uniformly distributed in the range of $\varphi$ $\in[0,2 \pi)$. We assume as fixed, for the rest of the paper, the values $\left(\theta_{0}, \alpha_{0}\right)=(0.2,0.2)$. The control parameters used were $\omega=1, \epsilon_{R}=\epsilon_{r}=0.01$, and the initial velocity was $v_{0}=2 \epsilon_{R}$ and in 9(a) and 9(c) $r=0.75$ and $d=0.05$; in 9(b) $r=0.15$ and $d$ $=0.65$. The average velocity grows since the beginning of the simulation up to $10^{7}$ iterations. For Fig. 9(a), the average velocity presents a hump at around $n \approx 10^{2}$ iterations. After that, the growth seems to be smooth and continuous. Figure 9(c) presents a zoom of Fig. 9(a) after $n=10^{2}$. We can therefore suppose that the average velocity grows according to

$$
\langle v\rangle \propto n^{\delta},
$$

where $\delta$ is the growth exponent. For Fig. 9(c) $(r=0.75$ and $d=0.05$ ), we obtained an exponent $\delta=0.1115(8)$; on the other hand, in Fig. 9(b) $(r=0.15$ and $d=0.65)$, we got $\delta$ $=0.393(3)$. We might therefore emphasize that all plots presented in Figs. 9(a)-9(c) show the Fermi acceleration.

For the previous results, the value considered for the initial velocity was $v_{0}=2 \epsilon_{R}$. Now we show in Fig. 10 the behavior of $\langle v\rangle \times n$ for four different values of $v_{0}$, namely: $v_{0}$ $=\left\{2 \epsilon_{R}, 10 \epsilon_{R}, 100 \epsilon_{R}, 1000 \epsilon_{R}\right\}$. The control parameters used were $r=0.15, d=0.65$ and (a) $\omega=1, \epsilon_{R}=\epsilon_{r}=0.01$; (b) $\epsilon_{R}$ $=0.01, \epsilon_{r}=\epsilon_{R}(\sqrt{5}+1) / 2$, and $\omega=(\sqrt{5}+1) / 2$. For low values of the initial velocity and after a very brief transient, the velocity of the particle presents a continuous growth, given by Eq. (30). However, as the initial energy increases, the average velocity remains constant along of a plateau spend- 

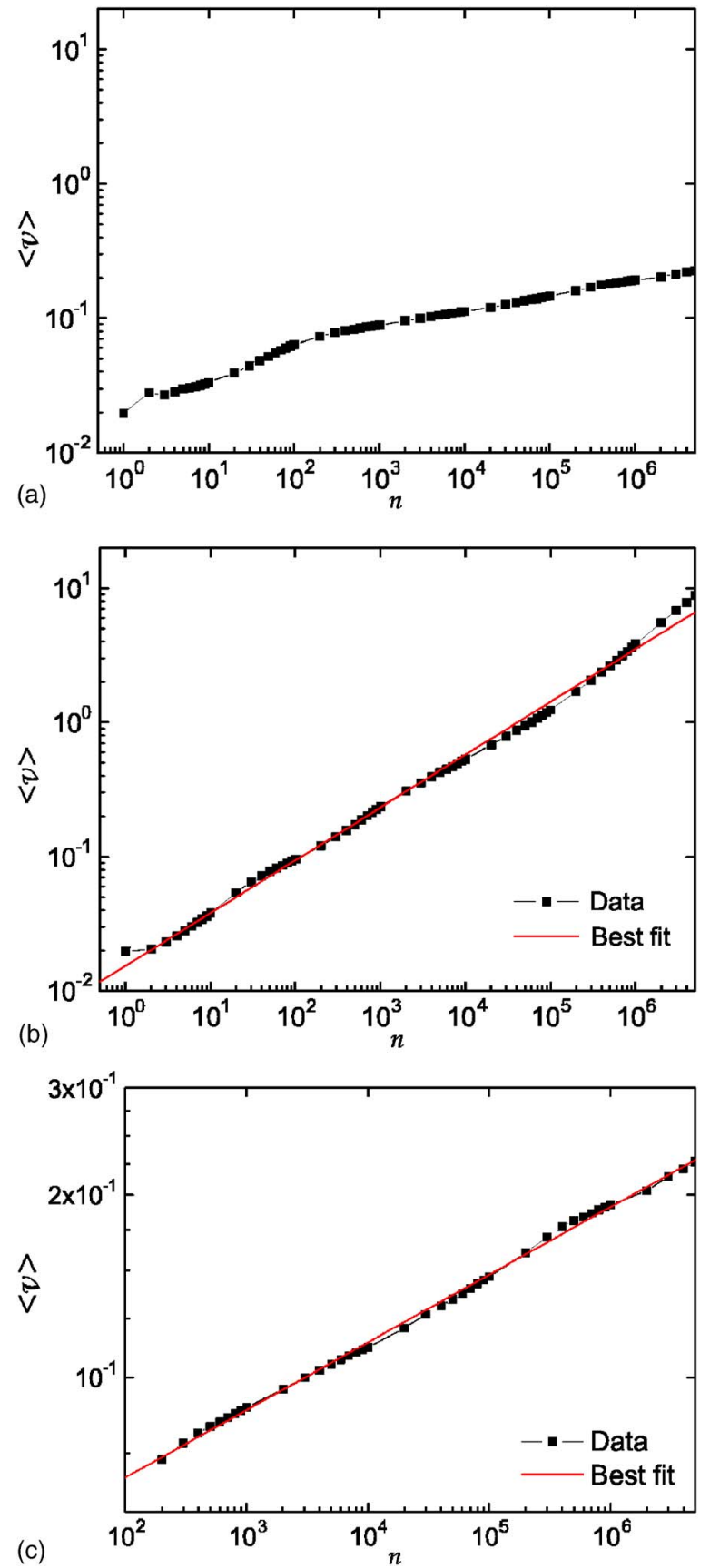

FIG. 9. (Color online) Log-log plot of the average velocity as function of the iteration number. The control parameters used were $\epsilon_{R}=\epsilon_{r}=0.01, v_{0}=2 \epsilon_{R}, \omega=1$, and (a) and (c) $r=0.75$ and $d=0.05$; (b) $r=0.15$ and $d=0.65$.

ing many iterations in a transient until changeover and bends towards a regime of growth. The transient depends sensitively on the initial velocity but all of them follow a growth envelope. For Fig. 10(a) the exponent is the same of that one obtained for Fig. 9(b), i.e, $\delta=0.393(3)$. A power law fitting for Fig. 10(b) gives $\delta=0.47(1)$. We emphasize that all the initial conditions given for the eccentric case with timevarying boundaries fell into the chaotic sea for the corresponding static version of the model. These results also give support to the LRA conjecture in the sense that the chaotic
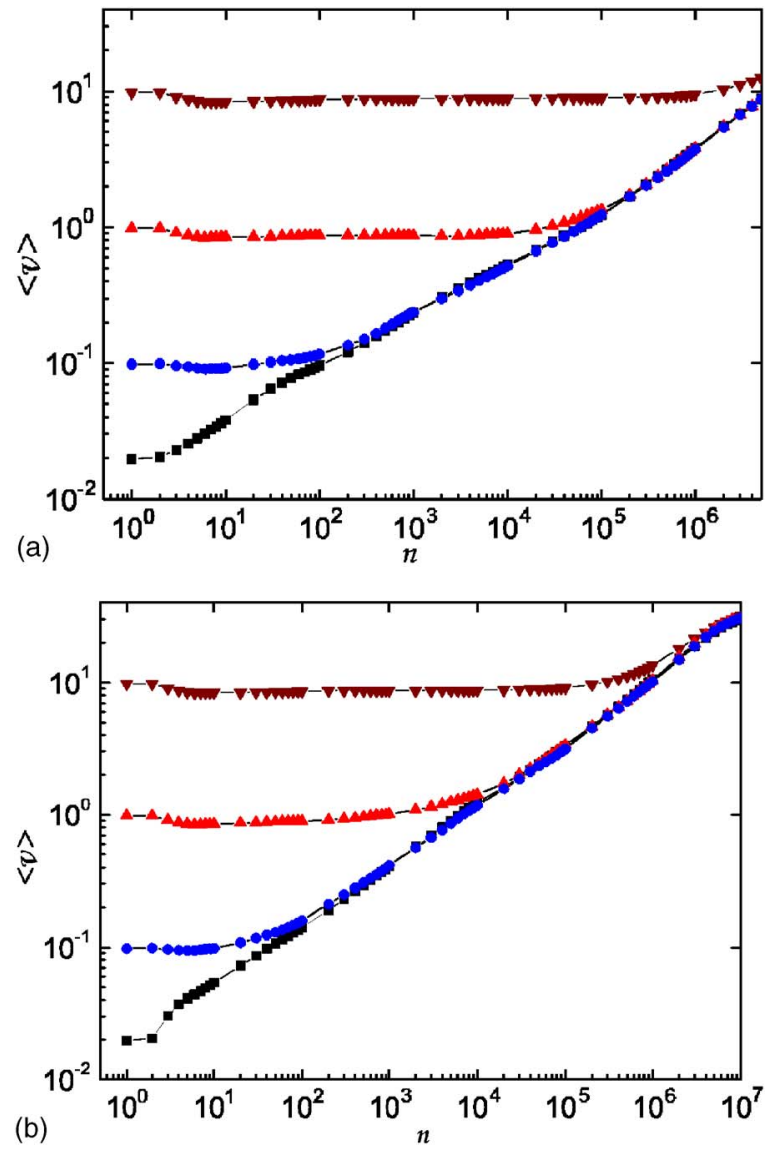

FIG. 10. (Color online) Log-log plot of the average velocity as function of the iteration number for different initial velocities: $v_{0}$ $=2 \epsilon_{R}, 10 \epsilon_{R}, 100 \epsilon_{R}, 1000 \epsilon_{R}$. The control parameters used were $r$ $=0.15, d=0.65$ and (a) $\epsilon_{R}=\epsilon_{r}=0.01, \omega=1$ and (b) $\epsilon_{R}=0.01, \epsilon_{r}$ $=\epsilon_{R}(\sqrt{5}+1) / 2, \omega=(\sqrt{5}+1) / 2$.

dynamics for the static version of the annular billiard implies in Fermi acceleration for the periodic time-dependent annular billiard.

\section{FINAL REMARKS AND CONCLUSIONS}

We have studied the annular billiard under two different situations: (i) static boundaries and (ii) periodic timedependent boundaries. The dynamics of the system was described using nonlinear maps. For case (i), the concentric annular billiard is globally integrable, while the eccentric annular billiard consists of a mixed system in the sense that resonance structures, chaotic orbits, and invariant spanning curves are either present in the phase space. In the case (ii) we derive, for the first time in this problem, a nonlinear discrete map that describes all the dynamics of the system. According to the LRA conjecture [21], if in the static case of a billiard system the dynamics is chaotic then one can observe Fermi acceleration. We have shown that the timedependent annular billiard does not present unlimited Fermi acceleration for the concentric case, which corroborates with the LRA conjecture. On the other hand, for the eccentric case the situation is very different since the static counterpart presents a chaotic dynamics. 
For the different combinations of control parameters which we have used in this paper, the periodic timedependent eccentric annular billiard presents unlimited energy growth. Particularly, we have shown that the average velocity grows according to a power law. Even though we did not exhaust all the dynamical scenarios of the model, with the set of parameters that we have selected we reinforce that the chaotic dynamics, in the static case, is a sufficient condition to observe Fermi acceleration. We would like to reinforce that the Fermi acceleration occurred in a scenario where the boundaries oscillate periodically and not stochastically, as reported in the majority of the works.

It is worthwhile to point out that analyzing the average velocity in terms of the collision number is a slightly different from analyzing it in terms of real time. This difference occurs because for low velocities the particle may suffer successive collisions with both boundaries when it goes into the collision zones of both boundaries. Consequently, it is expected that, in the average, the particle spends a long elapsed time until hitting again the external boundary. On the other hand, for intermediate and high velocities this problem is attenuated because the particle escapes faster from the collision zones in such a way that the average velocities are completely analogous if one computes the real time or the collision number. In order to minimize the fluctuations originated from the low velocities we generate a great ensemble of different initial conditions.

Finally, we also point out that this billiard might be considered as a transversal section of a three-dimensional toroidal setup of a particle accelerator. In this sense, our work supplies theoretical ideas and numerical results that could be used to accelerate particles in laboratories.

\section{ACKNOWLEDGMENT}

This research was partially supported by the scientific Brazilian agencies, Conselho Nacional de Desenvolvimento Científico e Tecnológico (CNPq), and Coordenação de Aperfeiçoamento de Pessoal de Nível Superior (CAPES).
[1] E. Fermi, Phys. Rev. 75, 1169 (1949).

[2] S. Ulam, in Proceedings of the 4th Berkeley Symposium on Math, Stat and Probability (University Press, Berkeley, CA, 1961), Vol. 3.

[3] G. M. Zaslavsky and B. V. Chirikov, Dokl. Akad. Nauk UzSSR 159, 306 (1964).

[4] A. J. Lichtenberg and M. A. Lieberman, Phys. Rev. A 5, 1852 (1972).

[5] A. Brahic, Astron. Astrophys. 12, 98 (1971).

[6] L. D. Pustylnikov, Theor. Math. Phys. 57, 1035 (1983).

[7] S. M. Ulam, Los Alamos, Report No. MS-2219, 1958.

[8] G. M. Zaslavisky, Chaos in Dynamical Systems (Harwood, Amsterdam, 1985).

[9] A. J. Lichtenberg, M. A. Lieberman, and R. H. Cohen, Physica D 1, 291 (1980).

[10] E. D. Leonel and J. K. L. da Silva, Physica A 323, 181 (2003).

[11] E. D. Leonel and P. V. E. McClintock, J. Phys. A 37, 8949 (2004).

[12] E. D. Leonel and P. V. E. McClintock, Phys. Rev. E 70, 016214 (2004).

[13] E. D. Leonel and P. V. E. McClintock, J. Phys. A 38, 823 (2005).

[14] A. Loskutov, A. B. Ryabov, and L. G. Akinshin, J. Exp. Theor. Phys. 89, 966 (1999).

[15] G. M. Zaslavsky, Phys. Rep. 371, 461 (2002).

[16] G. M. Zaslavsky, Chaos 4, 25 (1994).

[17] G. M. Zaslavsky, Physica D 76, 110 (1994).

[18] A. I. Saichev and G. M. Zaslavsky, Chaos 7, 753 (1997).

[19] J. L. Mateos, Phys. Rev. Lett. 84, 258 (2000).

[20] G. M. Zaslavsky, Chaos 15, 015103 (2005).

[21] A. Loskutov, A. B. Ryabov, and L. G. Akinshin, J. Phys. A 33,
7973 (2000).

[22] A. Loskutov and A. Ryabov, J. Stat. Phys. 108, 995 (2002).

[23] O. Bohigas, D. Boosé, R. Egydio de Carvalho, and V. Marvulle, Nucl. Phys. A 560, 197 (1993).

[24] R. Egydio de Carvalho and A. P. Mijolaro, Phys. Rev. E 70, 056212 (2004).

[25] R. Egydio de Carvalho, Phys. Rev. E 55, 3781 (1997).

[26] C. Dembowski, H.-D. Graff, A. Heine, R. Hofferbert, H. Rehfeld, and A. Richter, Phys. Rev. Lett. 84, 867 (2000).

[27] R. Hofferbert, H. Alt, C. Dembowski, H.-D. Graff, H. L. Harney, A. Heine, H. Rehfeld, and A. Richter, Phys. Rev. E 71, 046201 (2005).

[28] S. D. Frischat and E. Doron, Phys. Rev. E 57, 1421 (1998).

[29] E. Doron and S. D. Frischat, Phys. Rev. Lett. 75, 3661 (1995).

[30] R. Egydio de Carvalho, F. C. de Souza, and E. D. Leonel, J. Phys. A 39, 3561 (2006).

[31] N. Saitô, H. Hirooka, J. Ford, F. Vivaldi, and G. H. Walker, Physica D 5, 273 (1982).

[32] G. Gouesbet, S. Meunier-Guttin-Cluzel, and G. Gréhan, Phys. Rev. E 65, 016212 (2001).

[33] G. Gouesbet, S. Meunier-Guttin-Cluzel, and G. Gréhan, Opt. Commun. 201, 223 (2002).

[34] S. O. Kamphorst and S. P. Carvalho, Nonlinearity 12, 1363 (1999).

[35] A. J. Lichtenberg and M. A. Lieberman, Regular and Chaotic Dynamics, Applied Mathematical Sciences Series Vol. 38 (Springer-Verlag, New York, 1992).

[36] A. M. Ozorio de Almeida, Hamiltonian Systems: Chaos and Quantization (Cambridge University Press, Cambridge, MA, 1988). 